\title{
The fixed point theorem for simplicial nonpositive curvature
}

\author{
BY PIOTR PRZYTYCKI \\ Institute of Mathematics, Polish Academy of Sciences, Sniadeckich 8, \\ 00-956 Warsaw, Poland. \\ e-mail: pprzytyc@mimuw.edu.pl
}

(Received 21 December 2006; revised 15 May 2007)

Partially supported by MNiSW grant N201 003 32/0070

\begin{abstract}
We prove that for an action of a finite group $G$ on a systolic complex $X$ there exists a $G$-invariant subcomplex of $X$ of diameter $\leqslant 5$. For 7 -systolic locally finite complexes we prove there is a fixed point for the action of any finite $G$. This implies that free products with amalgamation (and HNN extensions) of 7-systolic groups over finite subgroups are also 7-systolic.
\end{abstract}

\section{Introduction}

Systolic complexes and systolic groups were introduced by T. Januszkiewicz and J. Świątkowski in [6] and independently by F. Haglund in [3]. Systolic complexes are simplyconnected simplicial complexes satisfying certain link conditions. Some of their properties are very similar to the properties of CAT(0) metric spaces, therefore one calls them complexes of simplicial nonpositive curvature. In particular it was shown in [6, theorem 4.1(1)], that they are contractible. One also has the notion of convexity.

For CAT(0) spaces we have the following fixed point theorem.

THEOREM $1 \cdot 1$ ([1, chapter II.2, corollary $2 \cdot 8])$. If $X$ is a complete CAT $(0)$ space and $G$ is a finite group of isometries then the fixed-point set of $G$ is non-empty.

Theorem $1 \cdot 1$ follows from an observation that for every bounded subset $Y$ of a CAT(0) space we can define a special point $y$, the circumcenter of $Y$, which is the center of the (unique) minimal ball containing $Y$. The circumcenter $y$ is invariant under isometries which leave $Y$ invariant.

For simplicial nonpositive curvature a minimal combinatorial ball containing finite set is not unique and the distance between centers of various minimal balls can be arbitrarily large. Thus there is no immediate way to define circumcenter.

We have found an analogue of Theorem 1.1 for actions of finite groups on systolic complexes. This paper is devoted to the proof of the following theorem.

THEOREM 1·2. Let $G$ be a finite group acting by simplicial automorphisms on a systolic complex $X$. Then there exists a subcomplex $Y \subset X$ which is invariant under the action of $G$ and whose diameter is $\leqslant 5$. 
This is a coarse version of the fixed point theorem. We use it to obtain in systolic setting the property, which for $\mathrm{CAT}(0)$ groups is implied by Theorem $1 \cdot 1$.

COROLlaRY 1.3. Any systolic group contains only finitely many conjugacy classes of finite subgroups.

The class of 7-systolic complexes is a special subclass of the class of systolic complexes. 7-systolic complexes are hyperbolic metric spaces ([6, theorem 2.1]) thus 7-systolic groups (defined as the groups which act properly and cocompactly on 7-systolic complexes) are hyperbolic. All hyperbolic groups have finitely many conjugacy classes of finite subgroups, so Corollary 1.3 is nothing new for 7 -systolic groups. However, for locally finite 7 -systolic complexes we can go further than Theorem 1.2 and obtain an honest fixed point theorem.

THEOREM 1.4. Let $G$ be a finite group acting by simplicial automorphisms on a locally finite 7-systolic complex $X$. Then there exists a simplex $\sigma \in X$, which is invariant under the action of $G$. (The barycenter of $\sigma$ is a fixed point for $G$.)

We apply Theorem 1.4 to show that the class of 7 -systolic complexes is closed under certain algebraic operations.

THEOREM 1.5. Free products of 7-systolic groups amalgamated over finite subgroups are 7-systolic. HNN extensions of 7-systolic groups over finite subgroups are 7-systolic.

The paper is organized in the following way. In Section 2 we recall the notion and properties of systolic complexes and groups. In Section 3 we introduce the notion of round complexes (which are an obstruction to finding the circumcenter) and study their properties. In Section 4 we obtain a weaker version of Theorem 1.2, which still implies Corollary 1.3. This weaker version is more elegant in proof and it serves as a demonstration of our method, which is fully applied in Section 5. In Section 6 we prove Theorem 1.4 and in Section 7 we apply it to prove Theorem 1.5.

\section{Systolic complexes}

Let us recall (from [6]) the definition of a systolic complex and a systolic group.

Definition 2.1. A subcomplex $K$ of a simplicial complex $X$ is called full in $X$ if any simplex of $X$ spanned by vertices of $K$ is a simplex of $K$. The span of a subcomplex $K \subset X$ is the smallest full subcomplex of $X$ containing $K$. We will denote it by $\operatorname{span}(K)$. A simplicial complex $X$ is called flag if any set of vertices, which are pairwise connected by edges of $X$, spans a simplex in $X$. A simplicial complex $X$ is called $k$-large, $k \geqslant 4$, if $X$ is flag and there are no embedded cycles of length $<k$, which are full subcomplexes of $X$ (i.e. $X$ is flag and every simplicial loop of length $<k$ and $\geqslant 4$ has a diagonal).

Definition 2.2. A simplicial complex $X$ is called systolic if it is connected, simply connected and links of all simplices in $X$ are 6-large. A group $\Gamma$ is called systolic if it acts cocompactly and properly by simplicial automorphisms on a systolic complex $X$. (Properly means $X$ is locally finite and for each compact subcomplex $K \subset X$ the set of $\gamma \in \Gamma$ such that $\gamma(K) \cap K \neq \varnothing$ is finite.) If the links of all simplices of $X$ are additionally $k$-large with $k \geqslant 6$ we call it (and the group) $k$-systolic. 
Recall [6, proposition 1.4], that systolic complexes are themselves 6-large. In particular they are flag. Moreover, connected and simply connected full subcomplexes of systolic (respectively $k$-systolic) complexes are themselves systolic (resp. $k$-systolic). It turns out a simplicial complex is $k$-systolic with $k \geqslant 6$ iff it is connected, simply connected and $k$-large.

Now we briefly treat the definitions and facts concerning convexity.

Definition 2.3. For every pair of subcomplexes (usually vertices) $A, B$ in a simplicial complex $X$ denote by $|A, B|(|A B|$ for vertices) the combinatorial distance between $A^{(0)}, B^{(0)}$ in $X^{(1)}$, the 1 -skeleton of $X$. A subcomplex $K$ of a simplicial complex $X$ is called 3-convex if it is a full subcomplex of $X$ and for every pair of edges $A B, B C$ such that $A, C \in K,|A C|=2$, we have $B \in K$. A subcomplex $K$ of a systolic complex $X$ is called convex if it is connected and links of all simplices in $K$ are 3-convex subcomplexes of links of those simplices in $X$.

In [6, lemma 7.2] authors conclude that convex subcomplexes of a systolic complex $X$ are contractible, full and 3-convex in $X$. For a subcomplex $Y \subset X, n \geqslant 0$, the combinatorial ball $B_{n}(Y)$ of radius $n$ around $Y$ is the span of $\left\{P \in X^{(0)}:|P, Y| \leqslant n\right\}$. (Similarly $S_{n}(Y)$ $=\operatorname{span}\left\{P \in X^{(0)}:|P, Y|=n\right\}$.) If $Y$ is convex (in particular, if $Y$ is a simplex) then $B_{n}(Y)$ is also convex, as proved in [6, corollary 7.5]. The intersection of a family of convex subcomplexes is convex and we can define the convex hull of any subcomplex $Y \subset X$ as the intersection of all convex subcomplexes of $X$ containing $Y$.

The paper [4] of F. Haglund and J. Świątkowski contains a proof of the following proposition, which will be used throughout the present paper.

Proposition 2.4 ([4, proposition 4.2]). A full subcomplex $Y$ of a systolic complex $X$ is convex if and only if $Y^{(1)}$ is geodesically convex in $X^{(1)}$ (i.e. if all geodesics in $X^{(1)}$ joining vertices of $Y$ lie in $\left.Y^{(1)}\right)$.

We will need a crucial projection lemma. The residue of a simplex $\sigma$ in $X$ is the union of all simplices in $X$, which contain $\sigma$.

LEMMA 2.5 ([6, lemma 7.7]). Let $Y$ be a convex subcomplex of a systolic complex $X$ and let $\sigma$ be a simplex in $B_{1}(Y)$ disjoint with $Y$. Then the intersection of the residue of $\sigma$ and of the complex $Y$ is a simplex (in particular it is nonempty).

Definition 2.6. The simplex as in Lemma 2.5 is called the projection of $\sigma$ onto $Y$.

Now let us recall some definitions and facts concerning flat minimal surfaces in systolic complexes proved by T. Elsner in [2].

Definition 2.7. The flat systolic plane is a systolic 2-complex obtained by equilaterally triangulating Euclidean plane. We denote it by $\mathbb{E}_{\Delta}^{2}$. A systolic disc is a systolic triangulation of a 2-disc and a flat disc is any systolic disc $\Delta$, which can be embedded into $\mathbb{E}_{\Delta}^{2}$, such that $\Delta^{(1)}$ is embedded isometrically into 1 -skeleton of $\mathbb{E}_{\Delta}^{2}$. A systolic disc $\Delta$ is called wide if $\partial \Delta$ is a full subcomplex of $\Delta$. For any vertex $v \in \Delta^{(0)}$ the defect of $v$ (denoted by $\operatorname{def}(v)$ ) is defined as $6-t(v)$ for $v \notin \partial \Delta$, and as $3-t(v)$ for $v \in \partial \Delta$, where $t(v)$ is the number of triangles in $\Delta^{(2)}$ containing $v$. It is clear that internal vertices of a systolic disc have nonpositive defects.

We will need the following easy and well-known fact. 
Lemma $2 \cdot 8$ (Gauss-Bonnet Lemma). If $\Delta$ is any triangulation of a 2 -disc, then

$$
\sum_{v \in \Delta^{(0)}} \operatorname{def}(v)=6
$$

Definition 2.9. Let $X$ be a systolic complex. Any simplicial map $S: \Delta \rightarrow X$, where $\Delta$ is a triangulation of a 2-disc, is called a surface. A surface $S$ is systolic, flat or wide if the disc $\Delta$ satisfies the corresponding property. If $S$ is injective on $\partial \Delta$ and minimal (the smallest number of triangles in $\Delta^{(2)}$ ) among surfaces with the given image of $\partial \Delta$, then $S$ is called minimal. A geodesic in $\Delta^{(1)}$ is called neat if it stays out of $\partial \Delta$ except possibly at its ends.

Lemma $2 \cdot 10$ ([2, lemma 2.5]). A systolic disc $D$ is flat if and only if it satisfies the following three condition:

(i) D has no internal vertices of defect $<0$;

(ii) $D$ has no boundary vertices of defect $<-1$;

(iii) any segment in $\partial D$ connecting vertices with defect $<0$ contains a vertex of defect $>0$.

THEOREM $2 \cdot 11$ ([2, theorem 3.1]). Let $X$ be a systolic complex. If $S$ is a wide flat minimal surface in $X$ then $S$ maps neat geodesics isometrically into $X^{(1)}$.

Finally, recall a powerful observation.

LEMMA 2.12. ([7, lemma 4.4]). Every full subcomplex of a systolic complex is aspherical.

\section{Round complexes}

Let us make some remarks to motivate the forthcoming definition.

\section{Remark 3.1.}

(1) If a finite group $G$ acts by simplicial automorphisms on a systolic complex, then there exists a bounded convex subcomplex $Y \subset X$ that is invariant under the action of $G$. To see this, take any vertex $x \in X$ and take the convex hull $Y$ of the set $G x=\{g(x): g \in G\}$. Since the set $G x$ is finite, the points of $G x$ are at distance $<d$ from $x$ for some finite $d$. Convexity of combinatorial balls implies that the points of $Y$ are also at distance $<d$ from $x$. The fact that $Y$ is invariant under the action of $G$ is immediate.

(2) Let $Y$ be a bounded systolic complex of diameter $d$ (by diameter we denote the maximal 1-skeleton distance $|V W|$ of vertices $V, W \in Y^{(0)}$ ) and let $G$ be a group acting on $Y$ by simplicial automorphisms. Then the convex subcomplex $\bigcap_{y \in Y^{(0)}} B_{d-1}(y)$ is invariant under the action of $G$ and its diameter is $\leqslant d-1$.

(3) This looks like a plan for getting invariant subcomplexes of arbitrarily small diameter. However, this plan is difficult to execute, since it is unclear how to exclude the possibility that $\bigcap_{y \in Y^{(0)}} B_{d-1}(y)$ is empty.

Definition 3.2. A bounded systolic complex $Y$ of diameter $d$ is called round if

$$
\bigcap_{y \in Y^{(0)}} B_{d-1}(y)=\varnothing \text {. }
$$

Note that this is equivalent to the property that for each vertex $V \in Y^{(0)}$ there is a vertex $W \in Y^{(0)}$ such that $|V W|=d$. 
We now start developing properties of round complexes, which will result in establishing a bound for their diameter.

LEMMA 3.3. Let $Y$ be a round complex of diameter $d$. Then there exists an edge $A B \in Y$ and vertices $V, W \in Y$ such that $|V B|=|W A|=d$ and $|V A|=|W B|=d-1$.

Proof. Fix any maximal simplex $\sigma \subset Y$. Let $k$ be the maximal number such that for each simplex $\sigma^{\prime} \subset \sigma$ of dimension $k$ there exists a vertex $V \in Y$ with $\left|V, \sigma^{\prime}\right|=d$. Note that since $Y$ is round we have $k \geqslant 0$. On the other hand if we denote by $n$ the dimension of $\sigma$ we have $k<n$, since if $\sigma$ would be at distance $d$ from some vertex $V$, then the projection of $\sigma$ onto $B_{d-1}(V)$ together with $\sigma$ would form a strictly greater simplex (c.f. Lemma 2.5 and Definition 2.6).

Take a simplex $\tau \subset \sigma$ of dimension $k+1$ for which there does not exist a vertex $V \in Y$ with $|V, \tau|=d$. Denote by $A, B$ any two vertices of $\tau$. Denote by $\tau_{A}, \tau_{B}$ faces of codimension 1 in $\tau$ not containing $A, B$ respectively. By definition of $k$ there exist vertices $V, W$ such that $\left|V, \tau_{A}\right|=\left|W, \tau_{B}\right|=d$. This implies $|V B|=|W A|=d$. The choice of $\tau$ implies $|V A|=|W B|=d-1$.

Definition 3.4. A $k$-hexagon is a subcomplex of $\mathbb{E}_{\Delta}^{2}$ obtained by taking $6 k^{2} 2$ dimensional simplices forming a regular hexagon of edge length $k$. A $k$-triangle is a subcomplex of $\mathbb{E}_{\Delta}^{2}$ obtained by taking $k^{2}$ 2-dimensional simplices forming a triangle of edge length $k$.

LEMMA 3.5. Let $k \geqslant 1$ be an integer and let $Y$ be a round complex of diameter $d \geqslant 3 k$. Then there exists a k-hexagon $H \subset Y$ whose 1-skeleton is isometrically emdedded in the 1 -skeleton $Y^{(1)}$.

Proof. Lemma 3.3 guarantees the existence of the vertices $A, B, V, W \in Y$ at appropriate distances.

Let $V^{\prime} \in Y$ be the furthermost vertex from $V$ which is common for some geodesics connecting $V$ to $A$ and $V$ to $W$. Then let $W^{\prime} \in Y$ be the furthermost vertex from $W$ which is common for some geodesics connecting $W$ to $B$ and $W$ to $V^{\prime}$. Take any loop obtained by concatenating some 1-skeleton geodesics connecting $A$ to $V^{\prime}, V^{\prime}$ to $W^{\prime}$ then $W^{\prime}$ to $B$ and then the edge $B A$. We claim that this loop does not have self-intersections. Indeed, the segments $A V^{\prime}$ and $B W^{\prime}$ do not intersect by the choice of $A, B, V, W$ (any geodesics $A V$ and $B W$ must be disjoint, since vertices on $A V$ are further from $B$ than from $A$ and vertices on $B W$ are nearer to $B$ that to $A$ ). The segment $V^{\prime} W^{\prime}$ does not intersect $A V^{\prime}$ (outside of $V^{\prime}$ ) by the choice of $V^{\prime}$. Finally, $V^{\prime} W^{\prime}$ does not intersect $B W^{\prime}$ (outside of $W^{\prime}$ ) by the choice of $W^{\prime}$.

Now among all surfaces, whose boundary is any such piecewise geodesic loop $A V^{\prime} W^{\prime} B A$ choose a surface $S: \Delta \rightarrow X$ of minimal area. Clearly, $S$ is a minimal surface. Moreover, since our loop is piecewise geodesic and these geodesics are chosen arbitrarily, the defect at every boundary vertex of $\triangle$ different from $A, B, V^{\prime}, W^{\prime}$ is at most 0 . Since the segments $A B$ and $B W^{\prime}$ form a geodesic, the defect at $B$ is at most 1, and similar argument shows the same for $A$. Gauss-Bonnet Lemma 2.8 implies now that the defects at $V^{\prime}$ and $W^{\prime}$ are equal to 2, at $A, B$ equal to 1 , at other boundary and interior vertices equal to 0 . Lemma 2.10 implies that $\Delta$ is flat. Analyzing possible subcomplexes of $\mathbb{E}_{\Delta}^{2}$ one easily sees that $\Delta$ has to be a trapezoid with sides $A B, V^{\prime} W^{\prime}$ parallel. 
Denote $d^{\prime}=\left|V^{\prime} W^{\prime}\right| \leqslant d$, so that the other edges of the trapezoid $\Delta$ have lengths $\left|A V^{\prime}\right|=$ $\left|B W^{\prime}\right|=d^{\prime}-1,|A B|=1$. Then $\left|V V^{\prime}\right|=|A V|-\left|A V^{\prime}\right|=(d-1)-\left(d^{\prime}-1\right)$ and similarly $\left|W W^{\prime}\right|=|B W|-\left|B W^{\prime}\right|=(d-1)-\left(d^{\prime}-1\right)$. Then the geodesic $V W$ has length $\left|V V^{\prime}\right|+\left|V^{\prime} W^{\prime}\right|+\left|W^{\prime} W\right|=2 d-d^{\prime}=d+\left(d-d^{\prime}\right) \geqslant d$. Since the diameter of $Y$ is $d$ we must have $d^{\prime}=d$, which implies $V=V^{\prime}$ and $W=W^{\prime}$.

Since $3 k \leqslant d$, there exists a $k$-hexagon $H \subset \Delta$. Let $T$ be a subcomplex obtained from $\Delta$ by deleting the two triangles containing $S^{-1}(V)$ and $S^{-1}(W)$. Then our surface $S$ restricted to $T$ is flat, wide and minimal. Therefore, by Elsner's Theorem $2 \cdot 11$, all neat geodesics in $T^{(1)}$ are mapped by $S$ onto geodesics in $Y^{(1)}$. If two vertices in $\mathrm{H}$ are not connected by a neat geodesic in T, then they lie on a boundary segment of $\Delta$, which we assumed at the beginning to be mapped isometrically. This implies that the 1-skeleton of the $k$-hexagon $H \subset T$ is mapped isometrically into $Y^{(1)}$. Identification of $H$ with $S(H)$ finishes the proof.

LEMMA 3.6. Let $Y$ be a bounded systolic complex of diameter $d$, and let $H \subset Y$ be a k-hexagon, whose 1-skeleton is isometrically embedded into $Y^{(1)}$. Let $V \in H$ be the vertex, which is the Euclidean center of $H$. Take $m$ such that $2 m \leqslant k$. Suppose that $W \in Y$ is a vertex at distance $d$ from $V$. Then there exists an m-hexagon $H^{\prime} \subset H$, such that $H^{\prime} \subset S_{d}(W)$.

Proof. Since $H^{(1)}$ is isometrically embedded in $Y^{(1)}$, the intersection $C=H \cap B_{d-1}(W)$ is convex in $H$ (Proposition 2.4). Denote by $S_{0}, \ldots, S_{5}$ the six $k$-triangles in $H$ whose vertices are $V$ and two consecutive vertices (in the Euclidean sense) of the boundary of $H$. Notice that since $V$ is not in $C$, then for each $i=0,1,2$ at least one of the opposite $S_{i}, S_{i+3}$ must have empty intersection with $C$. Moreover, if $C \cap S_{i} \neq \varnothing$ and $C \cap S_{i+2} \neq \varnothing$ (we treat indices $i=0, \ldots, 5$ modulo 6 ) then $C \cap S_{i+1} \neq \varnothing$. Together this implies that we have an $i$ such that $C \cap\left(S_{i} \cup S_{i+1} \cup S_{i+2}\right)=\varnothing$. We find the $m$-hexagon $H^{\prime}$ inside the union of these $k$-triangles.

COROLLARY 3.7. Let $Y$ be a round complex of diameter $d \geqslant 12$. Then there exists $a$ vertex $W$ and a 2-hexagon $H^{\prime} \subset Y$, whose 1-skeleton is isometrically embedded into $Y^{(1)}$ and such that $H^{\prime} \subset S_{d}(W)$.

Proof. We apply Lemma 3.5 to $Y$ and obtain a 4-hexagon $H$, whose 1-skeleton is isometrically embedded into $Y^{(1)}$. Let $V \in H$ be the vertex, which is the Euclidean center of $H$. Since $Y$ is round, there exists a vertex $W \in Y$ such that $|V W|=d$. Now applying Lemma 3.6 gives us the desired 2-hexagon $H^{\prime}$.

\section{The main argument}

Lemma 4.1. Let $W \in Y$ be a vertex in a systolic complex. Then for any sphere $S_{n}(W) \subset Y$ with $n \geqslant 1$ there is no 2-hexagon $H \subset S_{n}(W)$, whose 1-skeleton is isometrically embedded into $Y^{(1)}$.

Before we present the proof, we note that Corollary 3.7 and Lemma 4.1 immediately imply the following.

COROLLARY 4.2. The diameter of any round complex is $\leqslant 11$.

Proof of Lemma 4.1. We will prove the lemma by contradiction. Suppose there is a 2hexagon $H \subset S_{n}(W)$, whose 1-skeleton is isometrically embedded into $Y^{(1)}$. Then $n \geqslant 2$ (because the diameter of $H$ is 4 ). Denote by $A_{0}, A_{1} \ldots, A_{11}$ the vertices of the boundary of 
$H$ in their natural cyclic order. Now from the projections of the edges $A_{i} A_{i+1}$ (we treat $i$ 's modulo 12) onto $B_{n-1}(W)$ choose single vertices $B_{i}$. Denote $B=\operatorname{span}\left(\bigcup_{i} B_{i}\right)$. Note that for any $i$ the vertices $B_{i}, B_{i+1}$ are either equal or connected by an edge (this follows from projection Lemma 2.5).

Let us denote $D_{1}=\operatorname{span}(B \cup H), D_{2}=\operatorname{span}\left(B \cup B_{n-2}(W)\right)$. Observe first that $D_{1} \cup D_{2} \subset$ $Y$ is full because there are no edges between the complexes $H \subset S_{n}(W)$ and $B_{n-2}(W)$. We will use Meyer-Vietoris sequence for the pair $D_{1}, D_{2}$. Namely consider the loop $B_{0} B_{1} \cdots B_{11} B_{0}$, which is contractible both in $D_{1}$ and $D_{2}$ (which is clear). These contractions form a 2-sphere in $D_{1} \cup D_{2}$ which is contractible, as $D_{1} \cup D_{2}$ is aspherical (Lemma 2.12). Thus the existence (and the form) of the homomorphism $H_{2}\left(D_{1} \cup D_{2}\right) \rightarrow H_{1}\left(D_{1} \cap D_{2}\right)$ in the Meyer-Vietoris sequence implies $B_{0} B_{1} \cdots B_{11} B_{0}$ is homological to zero in $D_{1} \cap D_{2}=B$.

We will show this is not possible. Namely, we will construct a continuous mapping from $B$ into $\mathbb{R}^{2} \backslash\{0\}$ such that the loop $B_{0} B_{1} \cdots B_{11} B_{0}$ is mapped to a nontrivial loop. First we construct a map $f: B^{(0)} \rightarrow \mathbb{R}^{2}$. Denote $e_{k}=e^{2 \pi i \frac{k}{12}} \in \mathbb{C}=\mathbb{R}^{2}$. For each vertex of $B$ we choose any $i$ such that this vertex is equal to $B_{i}$ and we map it to $e_{i}$ (this mapping is not unique only if for some $i \neq j$ we have $B_{i}=B_{j}$ ). Then we extend linearly to all simplices of $B$. Let $\langle\cdot, \cdot\rangle$ denote the standard scalar product in $\mathbb{R}^{2}$. Fix a simplex $\sigma \subset B$ and suppose that $B_{i}, B_{j} \in \sigma$, where $i, j$ are the indices chosen when we defined $f$ on $B^{(0)}$. Since $\left|B_{i} B_{j}\right| \leqslant 1$ we have $\left|A_{i} A_{j}\right| \leqslant 3$, so $|i-j| \leqslant 3$ and thus $\left\langle f\left(B_{i}\right), f\left(B_{j}\right)\right\rangle \geqslant 0$. If we fix $i, j$ and consider some other $B_{k} \in \sigma$ (if $k$ is the index chosen for $B_{k}$ ) then the same observation yields that $f\left(B_{k}\right)$ belongs to $\left\{v:\|v\|^{2}=1,\left\langle v, f\left(B_{i}\right)\right\rangle \geqslant 0,\left\langle v, f\left(B_{j}\right)\right\rangle \geqslant 0\right\}$. The convex hull of this set omits 0 . This proves that the image of $f$ lies in $\mathbb{R}^{2} \backslash\{0\}$. Now observe that if $B_{i}=B_{j}$ for $i \neq$ $j$ (now the indices are arbitrary), then the distances $\left|A_{i} A_{j}\right|,\left|A_{i} A_{j+1}\right|,\left|A_{i+1} A_{j}\right|,\left|A_{i+1} A_{j+1}\right|$ are all $\leqslant 2$, which implies $|i-j|=1$. From this we see that the image under $f$ of each $B_{i}$ is $e_{i-1}, e_{i}$ or $e_{i+1}$. It follows that the image of each edge $B_{i} B_{i+1}$ lies in the sector between $e_{i-1}$ and $e_{i+2}$. This implies that the loop $B_{0} B_{1} \cdots B_{11} B_{0}$ is mapped to a nontrivial loop. Thus we have reached a contradiction.

Proof of Theorem 1.2 with bound $\leqslant 11$ instead of $\leqslant 5$. Let $Y \subset X$ be a bounded convex subcomplex invariant under the action of $G$, with the minimal possible (nonzero) diameter $d$. Such subcomplexes exist, as it follows from Remark 3.1(1). Now let $Y^{\prime}=$ $Y \cap\left(\bigcap_{y \in Y^{(0)}} B_{d-1}(y)\right)$. As it was noticed in Remark 3.1(2), the diameter of $Y^{\prime}$ is $<d$, so by minimality of the diameter of $Y, Y^{\prime}$ must be empty. Thus $Y$ is a round complex and by Corollary 4.2 its diameter is $\leqslant 11$.

Remark 4.3. For any bounded convex subcomplex $Y \subset X$ of a systolic complex $X$ we can define a sequence $Y=Y_{0}, Y_{1}, Y_{2}, \ldots$ by the formula $Y_{i+1}=Y_{i} \cap\left(\bigcap_{y \in Y_{i}^{(0)}} B_{d\left(Y_{i}\right)-1}(y)\right)$, where $d\left(Y_{i}\right)$ is the diameter of $Y_{i}$. The round complex on which this sequence terminates can be treated as the circumcenter of $Y$.

Proof of Corollary 1·3. We argue by contradiction. Suppose we have infinitely many conjugacy classes of finite subgroups represented by $H_{1}, H_{2}, \ldots \subset G$. Denote by $K \subset X$ the compact subset such that $\bigcup_{g \in G} g(K)=X$. For all $i \geqslant 1$ let $K_{i}$ be subcomplexes of $X$ with diameter $\leqslant 11$ invariant under $H_{i}$. Find $g_{i} \in G$ such that $g_{i}\left(K_{i}\right) \cap K \neq \varnothing$. Then the subgroups $g_{i} H_{i} g_{i}^{-1}$, which leave $g_{i}\left(K_{i}\right)$ invariant, still represent different conjugacy classes. In particular, the union $\bigcup_{i} g_{i} H_{i} g_{i}^{-1}$ is infinite. But for all elements $g$ of this union we have $g\left(B_{11}(K)\right) \cap B_{11}(K) \neq \varnothing$, which contradicts the properness of the action of $G$. 


\section{Round complexes have diameter $\leqslant 5$}

In this section we give the proof of even sharper bound for diameter of round complexes. It involves the same techniques, but takes considerably more case checking. First observe that as a special case of Lemma 3.5 we get the following.

COROLLARY 5.1. Let $Y$ be a round complex of diameter $d \geqslant 6$. Then there exists a 2-hexagon $H \subset Y$ whose 1-skeleton is isometrically emdedded in the 1-skeleton $Y^{(1)}$.

LEMMA 5.2. Let $Y$ be a bounded systolic complex of diameter d, and let $H \subset Y$ be a 2-hexagon, whose 1-skeleton is isometrically embedded into $Y^{(1)}$. Let $V \in H$ be the vertex, which is the Euclidean center of $H$. Suppose $W \in Y$ is a vertex at distance $d$ from $V$. Denote by $A_{0} A_{1} \cdots A_{11} A_{0}$ the boundary of $H$. Then there exists a simplicial loop $\gamma=$ $B_{0} B_{1} \cdots B_{11} B_{0}$ in $S_{d-1}(W)$, such that each $B_{i}$ is connected by an edge with $A_{i}$ and $A_{i+1}$ (or is equal to one of them) and $\gamma$ is contractible in $\operatorname{span}\left(\gamma \cup\left(H \cap S_{d}(W)\right)\right)$.

Proof. As in the proof of Lemma 3.6 we obtain three consecutive 2-triangles in $H$, say $S_{0}, S_{1}, S_{2}$, such that $S_{0} \cup S_{1} \cup S_{2} \subset S_{d}(W)$. Denote the boundary vertices of $H$ by $A_{0}, \ldots, A_{11}$ so that $A_{5}, A_{6}, \ldots, A_{11}$ lie in $S_{0} \cup S_{1} \cup S_{2}$. Pick vertices $B_{i}$ with $5 \leqslant i \leqslant 10$ from the projections of edges $A_{i} A_{i+1}$ onto $B_{d-1}(W)$.

Now we will pick $B_{4}$ and $B_{11}$. If $A_{0} \in S_{d}(W)$, then pick $B_{11}$ in the projection of $A_{11} A_{0}$ onto $B_{d-1}(W)$. In the other case pick $B_{11}=A_{0}$. Similarly pick $B_{4}$ from the projection of $A_{4} A_{5}$ if $A_{4} \in S_{d}(W)$ or pick $B_{4}=A_{4}$ in the other case.

Now we will pick $B_{0}$ and $B_{1}$. Denote by $U$ the vertex in $H$ which is the common neighbour of $A_{0}, A_{2}$ and $V$. Note that since $U$ is a neighbour of $V$, we have either $U \in S_{d-1}(W)$ or $U \in S_{d}(W)$. In the first case, pick $B_{0}=B_{1}=U$. In the second case proceed as follows. First we will pick $B_{0}$. Consider the edge $A_{0} A_{1}$. If it lies in $S_{d}(W)$, pick $B_{0}$ from the projection of $A_{0} A_{1}$ onto $B_{d-1}(W)$, as usual. If not, choose $B_{0}=A_{1}$ if $A_{1} \in S_{d-1}(W)$ and $B_{0}=A_{0}$ in the other case. Now we will pick $B_{1}$. If $A_{1} A_{2}$ lies in $S_{d}(W)$ then pick $B_{1}$ from the projection of $A_{1} A_{2}$, as usual. If not, choose $B_{1}=A_{2}$ if $A_{2}$ lies in $S_{d-1}(W)$ and $B_{1}=A_{1}$ in the other case.

Define $B_{3}, B_{2}$ exactly as $B_{0}, B_{1}$ substituting $B_{0}, B_{1}, A_{0}, A_{1}$ with $B_{3}, B_{2}, A_{4}, A_{3}$ respectively in the previous construction.

Note that our choice guarantees that for each $0 \leqslant i \leqslant 11$ the vertex $B_{i}$ is connected by an edge with $A_{i}$ and $A_{i+1}$ (or is equal to one of them).

First we will check that $B_{0} B_{11} \cdots B_{11} B_{0}$ is a simplicial loop, i.e. that $\left|B_{i} B_{i+1}\right| \leqslant 1$ for all $0 \leqslant i \leqslant 11$. For $4 \leqslant i \leqslant 10$ this follows from the projection Lemma $2 \cdot 5$. Now consider $i=11$. If $A_{0} \in S_{d}(W)$ then $\left|B_{11} B_{0}\right| \leqslant 1$ follows again from the projection Lemma $2 \cdot 5$. In the other case $B_{11}=A_{0}$, so it is also a neighbour of $B_{0}$. The analogous argument works for $i=3$.

Now focus on $i=0$. If $U \in S_{d-1}(W)$ (where $U$ is defined as in the construction of $\left.B_{0}, B_{1}\right)$, then $B_{0}=U=B_{1}$ and we are done. If not, then if $A_{1} \in S_{d}(W)$ then both $B_{0}, B_{1}$ are neighbours of $A_{1}$ in $S_{d-1}(W)$, so they are neighbours by Lemma 2.5. If $A_{1} \notin S_{d}(W)$, then $B_{0}=A_{1}$, so $B_{1}$ is its neighbour and we are also done. The analogous argument works for $i=1$.

Finally consider $i=2$. Define $U^{\prime}$ to be the common neighbour in $H$ of $A_{2}, A_{4}, V$. If both $U$ and $U^{\prime}$ are in $S_{d-1}(W)$, then $B_{1}=U, B_{2}=U^{\prime}$ and we are done. If any of $U, U^{\prime}$ lies in $S_{d-1}(W)$, then $A_{2}$ lies either in $S_{d}(W)$ or in $S_{d-1}(W)$. In the first case, both $B_{1}, B_{2}$ are neighbours of $A_{2}$ in $S_{d-1}(W)$, so they are connected by Lemma $2 \cdot 5$. In the second case $B_{1}=A_{2}=B_{2}$ and we are also done. 
We have thus proved that $B_{0} B_{1} \cdots B_{11} B_{0}$ is a simplicial loop, which we now denote by $\gamma$. We will prove that $\gamma$ is contractible in $\operatorname{span}\left(\gamma \cup\left(H \cap S_{d}(W)\right)\right)$. First observe that if the vertex $U$ (defined as before) is not in $H \cap S_{d}(W)$ then, by the construction of $B_{0}, U=B_{0}$ lies in $\gamma$. The same holds for $U^{\prime}$. So $S_{0} \cup S_{1} \cup S_{2} \cup U \cup U^{\prime} \subset \gamma \cup\left(H \cap S_{d}(W)\right.$. This is enough to guarantee that $\operatorname{span}\left(H \cap\left(\gamma \cup\left(H \cap S_{d}(W)\right)\right) \subset H\right.$ is contractible. Denote this subcomplex of $H$ by $H_{0}$.

Thus it is enough to prove that $\gamma$ can be homotoped into $H_{0}$ in $\operatorname{span}\left(\gamma \cup\left(H \cap S_{d}(W)\right)\right)$. If $\gamma$ is disjoint with $H_{0}$, then $\gamma$ is disjoint with $H$ and, by the construction of $\gamma$, we have $H_{0}=H \subset S_{d}(W)$. Hence, in this case, $\gamma$ is homotopic to $\partial H$ in $\operatorname{span}\left(\gamma \cup\left(H \cap S_{d}(W)\right)\right)$ (as in Lemma 4.1) and we are done. If $\gamma$ intersects $H_{0}$, then let $B_{i} B_{i+1} \cdots B_{j}$ denote any segment of $\gamma$ such that $B_{i}, B_{j} \in H_{0}$ and $B_{l} \notin H_{0}$ for all $i<l<j$ (with respect to the cyclic order). Then, by the construction of $B_{l}$, we have $A_{l} \in S_{d}$ for $i<l<j+1$, which implies $A_{l} \in H_{0}$. Thus the segment $B_{i} B_{i+1} \cdots B_{j}$ is homotopic (relative its endpoints) to the segment $B_{i} A_{i+1} A_{i+2} \cdots A_{j} B_{j} \subset H_{0}$ in $\operatorname{span}\left(\gamma \cup\left(H \cap S_{d}(W)\right)\right)$. Thus all segments of $\gamma$ lying outside $H_{0}$ can be homotoped into $H_{0}$ in $\operatorname{span}\left(\gamma \cup\left(H \cap S_{d}(W)\right)\right)$ and we are done.

\section{LEMMA 5·3. Every round complex has diameter $\leqslant 5$.}

Proof. Suppose, on the contrary, that $Y$ is a round complex of diameter $d \geqslant 6$. Then, by Corollary 5·1, we get a 2-hexagon $H \subset Y$ whose 1-skeleton is isometrically embedded in $Y^{(1)}$. Let $V \in H$ be the Euclidean center of $H$. Since $Y$ is round, there exists a vertex $W$ at distance $d$ from $V$. Denote by $A_{0} A_{1} \cdots A_{11} A_{0}$ the boundary of $H$. Then, by Lemma $5 \cdot 2$, there exists a loop $\gamma=B_{0} B_{1} \cdots B_{11} B_{0}$ in $S_{d-1}(W)$, such that $B_{i}$ is connected by an edge with $A_{i}$ and $A_{i+1}$ and $\gamma$ is contractible in span $\left(\gamma \cup\left(H \cap S_{d}(W)\right)\right)$. Let us denote $D_{1}=$ $\operatorname{span}\left(\gamma \cup\left(H \cap S_{d}(W)\right)\right), D_{2}=\operatorname{span}\left(\gamma \cup B_{n-2}(W)\right)$. Observe that $D_{1} \cup D_{2} \subset Y$ is full because there are no edges between the vertices in $S_{d}(W)$ and $B_{n-2}(W)$. Now we can proceed wordby-word following the proof of Lemma $4 \cdot 1$ and get a contradiction.

Proof of Theorem 1.2. As in the previous version of the proof in Section 4, we obtain an invariant convex subcomplex, which is round, so by Lemma $5 \cdot 3$ its diameter is $\leqslant 5$.

\section{Fixed point for 7-systolic complexes}

In this section we prove Theorem 1.4, the fixed point theorem for 7-systolic complexes (c.f. Definition 2.2). Notice that we already know that round 7-systolic complexes have diameter $\leqslant 2$, since for diameter $\geqslant 3$ Lemma 3.5 would produce a 1 -hexagon with 1 -skeleton isometrically embedded, which is not allowed in a 7-systolic complex. We can however skip this argument using a lemma by Osajda.

LeMma 6·1 ([5, lemma 3.1]). Suppose $|V Q|=|W Q|=n>0$ for some vertices $V, W, Q$ of a 7-systolic complex $X$. Denote by $P(V), P(W)$ the projections onto $B_{n-1}(Q)$ of $V, W$ respectively. Then either $P(V) \subset P(W)$ or $P(W) \subset P(V)$ (or both).

Proof of Theorem 1.4. Let $Y$ be a minimal (nonempty) connected and simply connected full subcomplex of $X$ invariant under $G$. By Remark 3.1(1) such subcomplexes exist. $Y$ is round by Remark 3.1(2). Moreover, $Y$ is 7-systolic, since it is a connected and simply connected full subcomplex of a 7-systolic complex $X$. We will show that $Y$ must be a simplex. Suppose on the contrary that $d=\operatorname{diam} Y \geqslant 2$.

We will show there exists a vertex $V \in Y$ such that for some vertex $W$ with $|V W|=1$ we have $B_{1}(V) \subsetneq B_{1}(W)$. In other words, (excluding $V$ and $W$ ) the set of neighbours of $W$ 
is strictly greater than the set of neighbours of $V$. One may then view $V$ as more exposed in $Y$ than $W$.

To prove this, pick any $Q \in Y$ and consider the family $\{P(V)\}_{V \in S_{d}(Q)}$ of all projections $P(V)$ onto $B_{d-1}(Q)$ of vertices in $V \in S_{d}(Q)$. Since $Y$ is round this family is nonempty. By projection Lemma 2.5 the elements of this family are simplices. Now consider a vertex $V$ such that $P(V)$ is minimal (for inclusion) simplex of the family. Take any vertex $W \in P(V)$. We will prove that $V, W$ have the desired property. Consider any neighbour $U$ of $V$. If $U \in S_{d-1}(Q)$, then $U \in P(V)$, so $U$ is a neighbour of $W$. If $U \in S_{d}(V)$, then by Lemma $6 \cdot 1$ and by minimality of $P(V)$ we get that $P(V) \subset P(U)$, hence $W \in P(U)$ and in this case also $U$ is a neighbour of $W$. Note that if we project $W$ onto $B_{d-2}(Q)$ (recall that $d \geqslant 2$ ) we obtain some neighbour of $W$, which is not a neighbour of $V$. This ends the proof of $B_{1}(V) \subsetneq B_{1}(W)$.

Notice that since the strict inclusion of 1-balls is a transitive relation and since $Y$ is finite, there exists a pair $|V W|=1, B_{1}(V) \subsetneq B_{1}(W)$, such that no neighbour $U$ of $V$ satisfies $B_{1}(U) \subsetneq B_{1}(V)$ (i.e. $V$ is a minimal element of this relation). Now consider the set $\mathcal{V}$ of all vertices $V \in Y$, which have the above minimality property. For each such vertex denote by $V^{\prime}$ its corresponding vertex $W$ (this choice may be not unique). Notice that for all $V \in \mathcal{V}$ we have $V^{\prime} \notin \mathcal{V}$, since $V^{\prime}$ cannot be minimal. We will now show that the subcomplex $Y^{\prime} \subset Y$ spanned on the vertices $Y^{(0)} \backslash \mathcal{V}$ is connected and simply connected. Since it is nonempty, invariant under $G$ and a strict subset of $Y$ this will contradict the minimality of $Y$ and will finish the proof.

To prove that $Y^{\prime}$ is connected and simply connected, we will construct a retraction $r: Y \rightarrow$ $Y^{\prime}$. First we define $r$ on $Y^{(0)}$. For $V \in Y^{\prime(0)}$ put $r(V)=V$. For $V \in \mathcal{V}$ put $r(V)=V^{\prime}$. We will prove that $r$ can be extended to a simplicial mapping. Since $Y^{\prime}$ is flag all we have to show is that for any adjacent vertices $V_{1}, V_{2} \in Y^{(1)}$ we have $\left|r\left(V_{1}\right) r\left(V_{2}\right)\right| \leqslant 1$. If $V_{1}, V_{2} \in Y^{\prime}$ then this is obvious. If $V_{1} \in Y^{\prime}, V_{2} \in \mathcal{V}$, then since $r\left(V_{1}\right)=V_{1}$ is a neighbour of $V_{2}$, it is also a neighbour of $r\left(V_{2}\right)$ and we are done. If $V_{1}, V_{2} \in \mathcal{V}$ then since $V_{1}$ is neighbour of $V_{2}$, it is also a neighbour of $r\left(V_{2}\right)$ and now since $r\left(V_{2}\right)$ is a neighbour of $V_{1}$, it is also a neighbour of $r\left(V_{1}\right)$ or equals $r\left(V_{1}\right)$ and we are done. Thus we can extend $r$ to a simplicial mapping $r: Y \rightarrow Y^{\prime}$ fixing $Y^{\prime}$ and thus, since $Y$ is connected and simply connected, so is $Y^{\prime}$. As observed earlier, this contradicts the minimality of $Y$. Thus $d \leqslant 1$ and $Y$ is a simplex.

Remark 6.2. We do not know if the assumption of Theorem 1.4 that $X$ is locally finite may be omitted.

\section{Amalgamated free products of 7-systolic groups}

We will prove Theorem 1.5 by constructing 7-systolic complexes on which the amalgamated products and HNN extensions act. These complexes will have a form of trees of 7-systolic complexes, as defined below, related to the Bass-Serre trees of the corresponding products.

Definition 7.1. A tree of $k$-systolic complexes $(k \geqslant 6)$ is a simplicial complex $E$ together with a simplicial mapping $p: E \rightarrow T$ onto a simplicial tree $T$ satisfying the following properties. For a vertex $V \in T$ the preimage $p^{-1}(V) \subset E$ is a $k$-systolic complex. For an open edge $e \in T$ the closure of the preimage $p^{-1}(e) \subset E$ is a simplex.

LEMMA 7.2. If $p: E \rightarrow T$ is a tree of $k$-systolic complexes, $k \geqslant 6$, then $E$ is itself $k$-systolic. 
Proof. To prove $E$ is $k$-systolic we need to prove $E$ is connected, simply connected and $k$-large (c.f. remarks after Definition 2.2). Obviously, $E$ is connected, simply connected and flag, since the preimage of each vertex and the closure of the preimage of each open edge in $T$ is flag and contractible and the same holds for their intersections. Let $\gamma$ be any loop of length $l$ with $4 \leqslant l<k$ in $E$. Then $p(\gamma)$ is a loop in the tree $T$. If $p(\gamma)$ is a single vertex, then $\gamma$ lies in a $k$-systolic subcomplex of $E$ and thus has a diagonal. If $p(\gamma)$ is not a vertex, then there exists two different edges $A B, A^{\prime} B^{\prime} \in \gamma$ such that $p(A B)=p\left(A^{\prime} B^{\prime}\right)$. This implies that $A, B, A^{\prime}, B^{\prime}$ lie in a common simplex. Since at least three of those vertices are different vertices of $\gamma$, we obtain a diagonal in the loop $\gamma$.

Construction 7.3. Let $G, H$ be 7-systolic groups acting properly and cocompactly on 7-systolic complexes $X, Y$, respectively. Let $F \subset G, F \subset H$ be some finite common subgroup. Let $\sigma \subset X, \tau \subset Y$ be some simplices fixed under $F$, as guaranteed by Theorem 1.4. We define the amalgamated complex $X * Y$ for $G *_{F} H$ as follows. Take the product space $G *_{F} H \times X \sqcup Y$ and identify $(a g, x)$ with $(a, g x)$ and $(a h, y)$ with $(a, h y)$ for all $a \in G *_{F} H, g \in G, h \in H, x \in X, y \in Y$. Note that this is an equivalence relation. As for now this is just a disjoint union of copies of $X$ and $Y$ corresponding to right cosets of $G$ and $H$ in $G *_{F} H$ respectively. Let $\alpha$ be an abstract simplex spanned on $\sigma$ and $\tau$ (the join of $\sigma$ and $\tau$ ). Extend the action of $F$ on $\sigma$ and $\tau$ to an affine (i.e. simplicial) action on $\alpha$. Now add extra simplicies $(a, \alpha)$ spanned on the pairs $(a, \sigma),(a, \tau)$ for $a \in G *_{F} H$ and identify $(a f, z)$ with $(a, f z)$ for $a \in G *_{F} H, f \in F, z \in \alpha$. Hence we added a copy of $\alpha$ for each coset $a F$ of $F$ in $G *_{F} H$. This copy of $\alpha$ is glued to the copies of $X$ and $Y$ corresponding to $a G$ and $a H$ respectively. Note that what we get is a simplicial complex, i.e. there are no multiple edges. The only multiple edges could occur as a result of gluing two copies of $\alpha$, say $(a, \alpha),(b, \alpha)$, where $a, b \in G *_{F} H$, to the same pair of copies of $X$ and $Y$. This would imply $(a, X)=(b, X)$ and $(a, Y)=(b, Y)$. Thus $b^{-1} a \in G \cap H=F$, hence $(a, \alpha)=(b, \alpha)$. Also note that since the action of $G$ on $X$ and $H$ on $Y$ is proper, the complex $X * Y$ is locally finite.

Now we define the action of $G *_{F} H$ on $X * Y$. Take $a, b \in G *_{F} H$ and $z \in X \sqcup Y$ or $z \in \alpha$. Define $a(b, z)=(a b, z)$. This is a simplicial automorphism of $X * Y$.

Construction 7.4. Let $G$ be a 7-systolic group acting properly and cocompactly on a 7systolic complex $X$. Let $F_{1}, F_{2}$ be some finite subgroups of $G$ isomorphic through a fixed isomorphism $i: F_{1} \rightarrow F_{2}$. Let $\sigma, \tau \subset X$ be some simplices fixed under $F_{1}, F_{2}$ respectively, as guaranteed by Theorem 1.4. We define the HNN extended complex $X *$ for $G *_{i}$ as follows. Denote by $t$ the element of $G *_{i}$ given in the presentation $t^{-1} f t=i(f), f \in F_{1}$. Take the product space $G *_{i} \times X$ and identify $(a g, x)$ with $(a, g x)$ for all $a \in G *_{i}, g \in G, x \in X$. Let $\alpha$ be an abstract simplex spanned on $\sigma$ and $\tau$ (treated as disjoint abstract simplices). Extend the action of $F_{1}$ on $\sigma$ and $\tau$ (on which $F_{1}$ acts as $F_{2}=i\left(F_{1}\right)$ ) to an affine (i.e. simplicial) action on $\alpha$. Now add extra simplicies $(a, \alpha)$ spanned on the pairs $(a, \sigma),(a t, \tau)$ for $a \in G *_{i}$ and identify $(a f, z)$ with $(a, f z)$ for $a \in G *_{i}, f \in F_{1}, z \in \alpha$. Again what we get is a simplicial complex. Loops cannot occur since $t \notin G \subset G *_{i}$ in the HNN extension and thus the copies of $X$ corresponding to cosets $a G$ and at $G$ are different. The only multiple edges could occur as a result of gluing two copies of $\alpha$, say $(a, \alpha),(b, \alpha)$, where $a, b \in G *_{i}$, to the same pair of copies of $X$. This would imply $(a, X)=(b, X)$ and $(a t, Y)=(b t, Y)$. Thus $b^{-1} a \in G \cap t G t^{-1}=F_{1}$, hence $(a, \alpha)=(b, \alpha)$. Since the action of $G$ on $X$ is proper, the complex $X *$ we obtained is locally finite. 
Now we define the action of $G *_{i}$ on $X *$. Take $a, b \in G *_{i}$ and $z \in X$ or $z \in \alpha$. Define $a(b, z)=(a b, z)$. This is a simplicial automorphism of $X *$.

LEMMA 7.5. Consider the complexes and groups acting on them from the Construction 7.3 and the Construction 7.4. The action of $G *_{F} H$ on $X * Y$ is proper and cocompact. The action of $G *_{i}$ on $X *$ is proper and cocompact.

Proof. We prove the first part of the lemma. Let $K_{X} \subset X$ and $K_{Y} \subset Y$ be compact sets, such that their translates through the elements of $G, H$ respectively fill in the corresponding complexes. Take $K \subset X * Y$ defined as $K=\left(1, K_{X}\right) \sqcup\left(1, K_{Y}\right) \sqcup(1, \alpha)$. The translates of $K$ through $G *_{F} H$ fill in $X * Y$, so the action is cocompact.

Now to prove the properness, since $X * Y$ is locally finite, it is enough to show that vertex stabilizers are finite. To do this, fix $b \in G *_{F} H, x \in X$, and suppose that $a(b, x)=(b, x)$ for some $a \in G *_{F} H$. Then there exists $g \in G$ such that $g x=x, a=b g b^{-1}$. Since $g$ determines $a$ and since the set of such $g$ is finite by the properness of the action of $G$ on $X$, the stabilizer of $(b, x)$ is finite. For $x \in Y$ the argument is the same.

The second part of the lemma can be proved in the same fashion.

LEMMA 7·6. Consider the complexes from the Construction $7 \cdot 3$ and the Construction 7.4. Then $X * Y$ and $X *$ are both trees of 7-systolic complexes.

Proof. We prove the first part of the lemma. Define a graph $T$ as follows. Let $V_{G}, V_{H}$ be right cosets of the subgroups $G, H$ in $G *_{F} H$. Let $V=V_{G} \sqcup V_{H}$ be the set of vertices of $T$. Let edges of $T$ be right cosets $a F$ of $F$ in $G *_{F} H$ joining the vertices $a G$ and $a H$. This graph is a tree, in fact it is the Bass-Serre tree of this amalgamated free product [8, special case of theorem 3.14]. Now define the simplicial mapping $p$ from $X * Y$ onto $T$. Define $p(a, x)=a G$ for $x \in X$ and $p(a, y)=a H$ for $y \in Y$. We can extend $p$ to a simplicial mapping. Then $p(a, \alpha)=a F$, where by $a F$ we mean the corresponding edge of $T$. From this construction it follows immediately that the closures of preimages of open edges in $T$ are simplices (the copies of $\alpha$ ) and that the preimages of vertices in $T$ are 7-systolic (these are copies of $X$ or $Y$ ).

For the second part, let $V$ be the set of the right cosets of $G$ in $G *_{i}$ and let edges be cosets $a F_{1}$ of $F_{1}$ in $G *_{i}$ joining the vertices $a G$ and $a t G$ in $V$. This graph $T$ is again a tree and we can define the simplicial mapping $p: X * \rightarrow T$ by $p(a, x)=a G$ for $a \in G * *_{i}, x \in X *$ and $p(a, \alpha)=a F_{1}$. As before, the preimages of vertices are 7-systolic copies of $X$ and the closures of preimages of open edges are simplices.

Proof of Theorem 1.5. Groups we consider act properly and cocompactly (Lemma 7.5) on trees of 7-systolic complexes (Lemma 7·6), which are 7-systolic by Lemma 7.2.

Remark 7.7. Using the same argument one can prove the following extension of Theorem $1 \cdot 5$. Let $k \geqslant 7$. Free products of $k$-systolic groups amalgamated over finite subgroups are $k$-systolic. HNN extensions of $k$-systolic groups over finite subgroups are $k$-systolic.

Remark 7·8. Note that the Constructions $7 \cdot 3$ and $7 \cdot 4$ work also for general (6-)systolic complexes whenever we amalgamate over groups which fix some simplices (for example if we amalgamate over the trivial group). We do not know if in general amalgamated products (and HNN extensions) of systolic groups over finite subgroups are systolic. 


\section{Final remarks on the general systolic case}

Remark 8.1. It seems that with current techniques we cannot get a sharper bound for the diameter of round complexes. We suspect, however, that round complexes have diameter $\leqslant 2$, because all round complexes we know have diameter $\leqslant 2$. An example of a round complex of diameter 2 is the 2 -triangle.

If it was true that round complexes have diameter $\leqslant 2$, we claim we could prove there is a fixed point for any simplicial action of a finite group on a locally finite systolic complex (and this would imply that amalgamates of systolic groups over finite subgroups are systolic). As a first step, we would find, like in the proof of Theorem 1.2, an invariant round complex, whose diameter would be $\leqslant 2$. Then we would use the following lemma that we proved together with P. Zawiślak.

LEMMA 8.2. In every finite systolic complex $Y$ of diameter $\leqslant 2$ there is a simplex $\sigma(Y)$, which is invariant under simplicial automorphisms of $Y$.

Note that we could have used Lemma 8.2 to prove Theorem 1.4. However, the proof of Theorem 1.4 we presented is simpler than the proof of Lemma 8.2. Since we treat Lemma $8 \cdot 2$ only as a digression, we do not enclose the proof.

Acknowledgements. I would like to thank Jacek Świa̧tkowski for posing the problem and for advice, Frederic Haglund for discussions, Paweł Zawiślak for developing the approach described in Remark 3.1 and the referee for valuable comments.

\section{REFERENCES}

[1] M. BRIDSON and A. HAEFLIGER. Metric Spaces of Non-Positive Curvature. Grundlehren der mathematischen Wissenschaften 319 (Springer, 1999).

[2] T. ELSNER. Flats and flat torus theorem in systolic spaces. Submitted.

[3] F. Haglund. Complexes simpliciaux hyperboliques de grande dimension. Preprint (Prepublication Orsay 71, 2003).

[4] F. Haglund and J. Świ ĄTKowski. Separating quasi-convex subgroups in 7-systolic groups. Groups, Geometry and Dynamics, to appear.

[5] D. OSAJDA. Ideal boundary of 7-systolic complexes and groups. Submitted.

[6] T. JANUSZKIEWICZ and J. ŚWI ĄTKOWSKI. Simplicial nonpositive curvature. Publ. Maths. Inst. Hanks Etudes Sci., 104(1) (2006), 1-85.

[7] T. JANUSZKIEWICZ and J. ŚWI ĄTKOWSKI. Filling invariants of systolic complexes and groups. Geom. Topol. 11 (2007), 727-758.

[8] P. SCOTT and T. WALL. Topological methods in group theory. In Homological Group Theory. London Math. Soc. Lecture Notes Ser. 36 (1979), 137-214. 